\title{
Apparent movement and metacontrast suppression: A decisional analysis
}

\author{
SEBASTIANO A. FISICARO, IRA H. BERNSTEIN, and PETER NARKIEWICZ \\ University of Texas at Arlington, Arlington, Texas 76010
}

\begin{abstract}
Three test and three mask energies were varied orthogonally and randomly over trials. The stimulus onset asynchrony (SOA) separating test and mask was varied between trial blocks within each of two display conditions, apparent movement (two-object) and metacontrast (threeobject). Subjects were required to make brightness judgments of both test and mask energies by responding "bright," "medium," or "dim" with respect to the apparent intensity of each stimulus. The accuracy and the coherence (consistency) of test judgments were U-shaped functions of SOA for both apparent movement and metacontrast situations. However, the accuracy and the coherence of mask judgments did not vary with SOA for either apparent movement or metacontrast. It was noted that substantially the same results have been reported previously when subjects were required to make contour judgments. Hence, it is argued that apparent movement and metacontrast suppression are intimately related.
\end{abstract}

Several theorists, such as Brunswik (see Hake, Rodwan, \& Weintraub, 1966), have proposed that the perceptual system is in essence a decision-making device. As such, the primary task of this system involves classification of multidimensional stimuli into perceptual categories. Quite often, the intent is to maximally discriminate between these categories. In an attempt to provide a model for perceptual judgments of this sort, Rodwan and Hake (1964) employed the linear discriminant function (LDF).

In this application, a stimulus comprised of $\mathrm{N}$ attributes or dimensions is considered a point in $\mathrm{N}$ space, with the axes delineating this space corresponding to the dimensions of stimulation. Concepts are defined by multivariate probability distributions and, thus, are viewed as regions in the $\mathrm{N}$-space. The means, or centroids, of these distributions correspond to the response categories. The classification of a particular observation into a response category is accomplished by considering the distances between the observation and each of the various centroids: the shorter the distance, the higher the probability that a particular response category is chosen.

Two manners in which these distances can be computed are as follows: (1) The subject can treat each dimension quasi-separately, compute the distance from the observation to the centroid along

Supported in part by a grant from the Organized Research Fund of the University of Texas at Arlington. The authors would like to thank Jan Stone for her help throughout the entire course of this experiment. A portion of these data was presented at the 1976 meeting of the Psychonomic Society, St. Louis, Missouri. Requests for reprints should be sent to Sebastiano A. Fisicaro, who is now at the Department of Psychology, Western Kentucky University, Bowling Green, Kentucky 42101. each of the dimensions of stimulation, and then combine these distances to infer the direct distance between the observation and the centroid; or (2) the subject can project the observation and the centroid onto a decisional axis in the $\mathrm{N}$-space and then compute the distance along this axis. The LDF is that decisional axis which represents the optimal weighting of the stimulus dimensions such that maximum discrimination between response categories is obtained by maximizing the betweencategory variance relative to the within-category variance. Hake, Faust, McIntyre, and Murray (1967) termed the former a discriminating observer model and the latter a nondiscriminating observer model.

Typically, in using the LDF to infer properties of the decisional structure, experiments are constructed such that some, if not all, of the dimensions of stimulation are relevant (to be responded to) and the remaining dimensions are irrelevant (to be ignored). For example, in the Hake et al. (1967) experiment, three inner and three outer square sizes were varied orthogonally to form nine stimulus alternatives. The experimental conditions were defined by the range of outer square variation relative to inner square variation. In all conditions, subjects were to classify the size of the inner square as "small," "medium," or "large." Thus, inner square and outer square sizes comprised the relevant and irrelevant dimensions, respectively, in forming a 2-space.

Application of the LDF to data obtained from experiments such as the one just described allows the calculation of certain performance measures. The coherence, or systematicity of responding, can be obtained by computing the separation among the centroids as projected onto the LDF, independently of accuracy, or separation among the centroids as 
projected onto the relevant axis of interest. Additionally, it is possible to distinguish between two sources of judgmental error. In one case, the subject's usage of information is inappropriate, whereas in the other case the subject's information is inadequate. Inappropriate responding occurs when the LDF is not parallel to the relevant axis (i.e., when it projects onto one or more of the irrelevant axes). Inadequate responding is inferred when there is a decrease in coherence from some appropriately chosen baseline. Since these two sources of error are not mutually exclusive, either or both forms may be found.

Recently, Bernstein, Fisicaro, and Fox (1976) applied the LDF to the study of metacontrast suppression. They noted that a subject may respond to different aspects of the stimulus environment as a particular variable is manipulated. Thus, they sought to infer the subject's criterion content, defined by Kahneman (1968) as a "description of the code that a subject uses in mapping his private experience onto responses to the experimenter's question"' (p. 410). Since the LDF describes the relative contributions of several sources of stimulus variation, Bernstein et al. (1976) viewed the LDF as a partial definition of criterion content.

In their study, three luminances were used for both test (center square) and mask (flanking squares) which, when varied orthogonally, formed nine stimulus alternatives. The relevant dimension was the energy of the test, and the irrelevant dimension was the energy of the mask. Seven SOAs were used to define the experimental conditions. The subject's task was to classify the test as "bright," "medium," or "dim" in attempting to maximize accuracy.

Bernstein et al. (1976) found that both the accuracy and the coherence of judgments were U-shaped functions of SOA, indicating that maximum suppression occurred when the mask was delayed and that metacontrast suppression is in part due to inadequate information. Furthermore, the subjects' criterion content was a combination of both test and mask energies, indicating that metacontrast is also accompanied by inappropriate responding. However, the form of this combination varied with SOA: The LDF had a negative slope at short SOAs but a positive slope at long SOAs. Thus, subjects responded to the test-mask energy difference at short SOAs but responded to the test-mask energy sum at long SOAs. It was noted that neurophysiological models of metacontrast which postulate that suppression results from lateral-inhibitory processes (e.g., Breitmeyer \& Ganz, 1976; Weisstein, 1968, 1972; Weisstein, Ozog, \& Szoc, 1975) cannot account for the positive correlation between mask energy and judgments of the test found at long SOAs.

A more cognitive approach has been taken by
Kahneman (1967) in attempting to explain metacontrast suppression. He noted that the mask in a metacontrast display produces conflicting apparentmovement cues. That is, in the case of the threeobject metacontrast display (center square and two flanking squares), the test appears to move in opposing directions. Perceived motion of this sort is physically impossible. It is therefore partially suppressed by the perceptual system, resulting in reduced visibility of the test. Thus, according to Kahneman (1967), metacontrast is anomalous stroboscopic motion.

Weisstein and Growney (1969) investigated the relationship between apparent motion and metacontrast. In a series of four experiments, they varied luminance level, stimulus duration, visual angle separating test and mask, and test-mask temporal asynchrony. The three-object display was used for metacontrast. By deleting one of the two flanking squares, a two-object display resulted, and this was used for apparent motion. The subject's task was to rate the "goodness" of movement in the two-object display and the "effectiveness" of metacontrast in the three-object display on a scale of 0 to 5 .

Briefly, the primary findings of Weisstein and Growney's (1969) study are as follows: Both the amplitude and the shape of the metacontrast function changed with visual angle, while the apparentmotion function remained relatively invariant. Furthermore, the amount of metacontrast suppression decreased at long test-mask asynchronies but the goodness of movement did not. Since these two psychological effects did not exhibit identical spatiotemporal patterns, Weisstein and Growney (1969) concluded that apparent motion does not provide a sufficient explanation of metacontrast.

In an attempt to account for their empirical observations, Weisstein and Growney (1969) reinterated Weisstein's (1968) previous claim that metacontrast suppression is a product of inhibitory processes. However, they attributed movement to the existence of single-unit motion detectors which fire when two short bursts of energy are summed. Thus, "the appearance of motion implies a lack of inhibition" (p. 328, italics added).

Breitmeyer, Love, and Wepman (1974) have also investigated the relationship between stroboscopic motion and metacontrast suppression. However, their study differed from that of Weisstein and Growney (1969) primarily as follows: (1) an accuracy task was used instead of a rating task; (2) contour judgments were required instead of brightness judgments; and (3) intercontour distance was not varied.

The subject's task was to verbally report whether two disks were "same" (both complete circles or both having a contour deletion) or "different" (one complete circle and one having a contour deletion). 
In the apparent-movement condition, only the two disks were presented but the temporal delay between them was varied. In the metacontrast condition, the two disks were presented simultaneously but each was followed at various temporal delays by an annulus (masking stimulus).

In both conditions, accuracy was a U-shaped function of temporal delay, and furthermore, these curves were indistinguishable. Breitmeyer et al. (1974) thus proposed that apparent movement and metacontrast contour suppression "may share common mechanisms"' (p. 1454).

However, Breitmeyer, Battaglia, and Weber (1976) noted that it was impossible to determine from the Breitmeyer et al. (1974) study whether the presentation of the first disk resulted in the suppression of information contained in the second disk or vice versa. Thus, they employed the apparentmovement display and used stimuli similar to those used by Breitmeyer et al. (1974) but required subjects to make separate judgments of the two disks by verbally responding "complete" or "deleted" to each. The results were that accuracy in judging the presence or absence of a contour deletion in the first stimulus (test) was a U-shaped function of SOA, but accuracy in judging the second stimulus (mask) was relatively high and did not vary with SOA. Breitmeyer et al. (1976) thus concluded that the second stimulus was suppressing information contained in the first stimulus.

Even though the different results obtained by Breitmeyer et al. $(1974,1976)$ and Weisstein and Growney (1969) may be attributed to the different judgments required of subjects (i.e., brightness vs. contour), they may also have arisen from the use of different tasks (i.e., rating vs. accuracy). Thus, the present experiment is an initial attempt at resolving this issue.

Several considerations resulted in a decision to use an accuracy task and require subjects to make separate brightness judgments about test energy and mask energy for both a two-object apparentmovement display and a three-object metacontrast display. An accuracy task was selected instead of a rating task for two basic reasons: First, it is an index of performance; and second, it lends itself more readily to an LDF analysis, which in turn allows the structure underlying judgments to be examined. Brightness judgments were chosen so that accuracy functions could be compared to the rating functions obtained by Weisstein and Growney (1969) and in order to replicate the Bernstein et al. (1976) findings. Judgments were required about both test and mask energies so that brightness accuracy functions could be compared to the contour accuracy functions obtained by Breitmeyer, Battaglia, and Weber (1976) and Breitmeyer, Love, and Wepman (1974).

\section{METHOD}

\section{Subjects}

Three graduate students and one undergraduate were paid $\$ 2.30 / \mathrm{h}$ to serve as subjects. All had either normal or correctedto-normal eyesight.

\section{Apparatus and Stimuli}

Stimuli were presented by transillumination on a four-channel Iconix tachistoscope (Model 6192) using Sylvania bulbs (F8TS/D). Viewing was monocular through a $2-\mathrm{mm}$ artificial pupil. A Spectra spotmeter (Model UBD-1) was used to verify luminance levels, and a Tektronix oscilloscope (Model 564) was used to verify timing accuracy. A telegraph key was used in order for subjects to self-initiate trials after both experimenters indicated that the necessary adjustments had been made. The two experimenters and the subject sat in the same room.

A small circle that remained on continually was used for fixation. It was contained in Exposure Field IV but illuminated by a separate tungsten source which did not affect the other contents of the field. The fixation circle was sufficiently dim so as to be barely visible to a light-adapted subject. It became clear with dark adaptation and, for this reason, served as a partial control over adaptation effects.

Exposure Field I was used for the three test stimuli (TS)-dim TS, medium TS, and bright TS. Each square appeared $.51^{\circ}$ of visual angle above the fixation circle. Exposure Fields II, III, and IV housed the three masking stimuli (MS) appropriate for the particular display. In the case of metacontrast, each MS consisted of a pair of flanking squares whose sides were adjacent to the TS. The movement MS were constructed merely by deleting one of the two metacontrast MS squares. ${ }^{1}$ For two subjects, the left square was deleted (rightward movement) and for the other two subjects the right square was deleted (leftward movement). The length of each side of TS and MS was $1.0^{\circ}$ of visual angle. The location of each MS with respect to the three fields used to house them was changed during the experiment so that each MS appeared equally often in each exposure field. This was done to eliminate coloration and other cues specific to each field that would provide information.

The TS and MS durations were both $50 \mathrm{msec}$. The three TS were changed manually by one of the two experimenters by dropping Kodak neutral density filters into Exposure Field 1. The other experimenter both selected the MS appropriate for that trial by means of a toggle switch, which enabled one of the three exposure fields (II, III, or IV) to fire, and recorded the subject's responses on an answer sheet. Luminances for bright TS and MS, medium TS and MS, and dim TS and MS were 64, 40, and $25 \mathrm{~cd} / \mathrm{m}^{2}$, respectively (i.e., $0,-.2$, and $-.4 \mathrm{log}$ units referenced to bright TS and MS).

\section{Procedure}

The subjects first participated in several practice sessions, during which they judged the brightness of the three TS energies alone (i.e., no MS) until their accuracy had apparently reached asymptote. The accuracies in terms of percent correct responses at the end of practice ranged from $56 \%$ to $75 \%$ and were within binomial error of one another for two consecutive sessions. At the beginning of each experimental session, the subjects were dark-adapted for $10 \mathrm{~min}$, after which they received 20-30 warm-up trials on TS alone with feedback. These data were not used further.

During the experiment, each subject received 270 trials at each of five SOAs $(0,30,60,120$, and $240 \mathrm{msec})$ under both movement (two-object) and metacontrast (three-object) displays, comprising the 10 experimental conditions. Additionally, 90 trials were run on TS alone and on metacontrast MS alone, comprising the two control conditions. No trials were run on movement MS alone, since this is essentially the same as TS alone.

The 270 experimental trials per SOA were divided into three 
90-trial blocks. Within each experimental block, each of the nine stimulus alternatives ( 3 TS energies $\times 3$ MS energies) appeared 10 times. This was done for both apparent movement and metacontrast conditions. Likewise, the 90 control trials of each type (TS alone and MS alone) were divided into three 30-trial blocks. Within each control block, each of the three TS (or MS) energies appeared 10 times. One experimental block per SOA was selected and these were randomly combined to form a cycle of five blocks. This process was repeated to form five more cycles. Thus, there were three movement cycles and three metacontrast cycles. In each of the six 1-h experimental sessions, the subject received one of the six cycles and one of the six control blocks. Different randomizations were used within each cycle and across subjects. Two subjects received the three movement cycles before the three metacontrast cycles. The reverse was true for the other two subjects.

The experimenters began each trial with appropriate switch adjustments and filter drops, including dummy switch adjustments and filter drops to avoid giving information to the subject. One experimenter then signaled the subject to initiate the trial, whereupon the subject responded "bright-bright," "brightmedium," "bright-dim," "medium-bright," etc., with respect to the apparent intensities of the TS-MS combination. Pilot data obtained from the present subjects indicated that the order of reporting concurrent test and mask judgments had no effect on any of the measures derived from the LDF analysis. Thus, subjects were instructed to report test judgments first. A short rest break was given after each block, during which one experimenter set the dials for the next SOA.

\section{RESULTS}

The discriminant functions were determined in the manner described by Hake et al. (1967) for each of 80 cases ( 2 Judgments by 2 Displays by 5 SOAs by 4 Subjects) using log energy as the metric. The first 40 cells ( 2 Displays by 5 SOAs by 4 Subjects) were constructed by analyzing judgments of the test (i.e., ignoring mask judgments) with TS energy considered as the relevant dimension and MS energy considered as the irrelevant dimension. The remaining 40 cells were obtained by analyzing judgments of the mask (i.e., ignoring test judgments) with MS energy considered as the relevant dimension and TS energy considered as the irrelevant dimension. The quantities needed to generate the LDFs for a given cell are the bivariate response centroids for the three response categories, the within-response-class sumof-products matrix (W), and the between-responseclass sum-of-products matrix (B). Two LDFs are obtainable from each cell, corresponding to the two eigenvectors of $\mathbf{W}^{-1} \cdot \mathrm{B}$. The first LDF accounted for at least $95 \%$ of the variance in 76 of the 80 cases. The second LDF never accounted for more than $22 \%$ of the variance in any case. This indicates that the centroids may be regarded as being colinear in the discriminant space.

Additionally, the analysis included univariate $F$ ratios for the two dimensions taken separately to determine whether or not the LDF differs significantly from being vertical (responses made solely to the relevant dimension) or horizontal (responses made solely to the irrelevant dimension). The relevant dimension contributed significantly beyond the .01 level $(\mathrm{df}=2,267)$ in all 80 cases. The irrelevant dimension contributed significantly beyond the .01 level ( $\mathrm{df}=2,267$ ) in 39 cases and beyond the .05 level in an additional 5 cases. The irrelevant dimension failed to contribute significantly in 7 (4 vs. 3) cases at 0 -msec SOA, 5 ( 3 vs. 2 ) cases at 30 msec SOA, 11 (6 vs. 5) cases at 60-msec SOA, 5 ( 3 vs. 2 ) cases at $120-\mathrm{msec}$ SOA, and 8 (4 vs. 4 ) cases at $240-\mathrm{msec}$ SOA. The numbers in parentheses represent the nonsignificant cases partitioned into metacontrast vs. apparent movement, respectively. As can be seen, the two displays produced very similar (if not identical) temporal patterns in terms of their influence on subjects' judgments. Furthermore, the criterion content of the judgments was some combination of both relevant and irrelevant dimensions, indicating that both metacontrast and apparent-motion displays produce inappropriate responding.

However, in order to determine the manner in which information about the two dimensions was combined, it is necessary to examine the slopes of the LDFs. These slopes (expressed in terms of the angle between the LDF and the relevant axis) are depicted in Figure 1. Judgments of the test (left panel) will be considered first. The results obtained with the metacontrast display are identical to those reported by Bernstein et al. (1976): Slopes of the LDFs are negative at short (0- and $30-\mathrm{msec})$ SOAs, indicating that high mask energies led to "dim" responses and that subject reacted to the test-mask energy difference. Additionally, slopes of the LDFs are positive at long (60- and $120-\mathrm{msec})$ SOAs, indicating that high mask energies led to "bright" responses and that subjects reacted to the test-mask energy sum. The return to zero at $240-\mathrm{msec}$ SOA indicates that the LDFs were essentially parallel to the relevant axis. In other words, subjects were responding solely to the energy of the test while ignoring the mask. This was expected on the basis

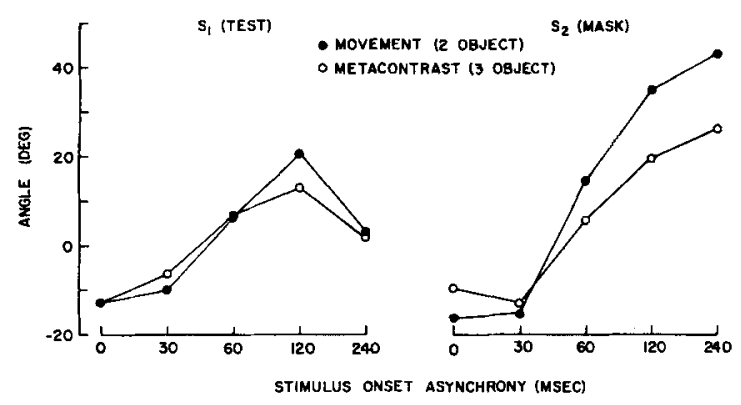

Figure 1. Slope of LDF (expressed in terms of the angle, in degrees, between the LDF and the relevant axis) for the two displays as a function of SOA for judgments of the test (left panel) and mask (right panel). 
of previous findings (Bernstein et al., 1976) that a 180 -msec delay in mask presentation is sufficiently long to allow subjects to make absolute judgments about the test.

The most interesting aspect of the data is that the slopes obtained with the apparent-movement display are the same as those obtained with the metacontrast display. A within-subjects analysis of variance (2 Displays by 5 SOAs by 4 Subjects) produced the following: There was no main effect of Display, $F(1,3)$ $<1.0$, MSe $=23.89$, a significant main effect of SOA, $\mathrm{F}(4,12)=6.31, \mathrm{p}<.01$, MSe $=176.99$, and no Display by SOA interaction, $F(4,12)<1.0$, $\mathrm{MSe}=56.32$.

It should be noted that the negative correlations found at short SOAs are explicable in terms of lateral inhibition, but the positive correlations found at long SOAs are not. Furthermore, while Weisstein and Growney's (1969) contention that apparent motion implies disinhibition is consistent with the data obtained at long SOAs, it is clearly not supported by the data obtained at short SOAs.

As for judgments of the mask (right panel), again the data obtained under the two displays are the same. The analysis of variance indicated no main effect of Display, $F(1,3)<1.0, \mathrm{MSe}=716.27$, a significant main effect of SOA, F(4,12) $=13.60, p<.01$, MSe $=297.17$, and no Display by SOA interaction, $\mathrm{F}(4,12)=1.21, \mathrm{p}>.05, \mathrm{MSe}=180.65$. The apparent divergence of the curves at 120- and $240-\mathrm{msec}$ SOA is due to two of the four subjects.

Except for the two large positive slopes obtained with the movement display at the two longest SOAs, the data points for mask judgments span a range similar to that observed for judgments of the test. The primary difference between the two sets of functions is that the mask-judgment curves do not return to zero at 240 -msec SOA. This means that subjects' judgments of the mask were still being influenced by the decaying trace of the test. Thus, with longer SOAs these curves would be expected to drop.

The accuracy of the judgments at a given SOA in a particular condition is defined as the ratio of the between-groups sum-of-squares to the within-groups sum-of-squares measured along the relevant axis. It is computed from the quadratic form of $W^{-1} \cdot B$ weighted by the vector $[1,0]$. Likewise, the coherence, or systematicity of responding, is defined as the corresponding ratio measured along the discriminant. It is obtained from the quadratic form of $\mathrm{W}^{-1} \cdot \mathrm{B}$ weighted by the LDF. Figure 2 contains the mean accuracy over subjects for the two displays as a function of SOA separately for test judgments (left panel) and mask judgments (right panel). Also included are the mean accuracies inferred from the two control conditions: test only (i.e., no mask) and mask only (i.e., no test). Figure 3 contains the comparable data for the coherence measure.

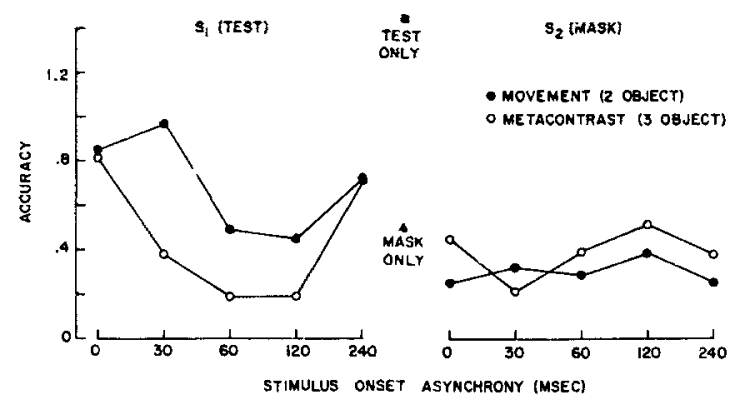

Figure 2. Accuracy (expressed in terms of the quadratic form of $W^{-1} \cdot B$ weighted by the vector $\left.[1,0]\right)$ for the two displays as a function of SOA for judgments of the test (left panel) and mask (right panel). Also included are the accuracy in judging the test alone (i.e., no mask) and the accuracy in judging the mask alone (i.e., no test).

Separate within-subjects analyses ( 2 Displays by 5 SOAs by 4 Subjects) were performed on the accuracies of test judgments and mask judgments. The analysis of test judgments resulted in a main effect of SOA, $F(4,12)=12.83, p<.01$, MSe $=$ .03 , no main effect of Display, $F(1,3)=5.39$, $\mathrm{p}>.05$, MSe $=.11$, and no Display by SOA interaction, $F(4,12)=2.78, p>.05$, MSe $=.04$. However, a Scheffé post hoc analysis demonstrated a significant difference $(p<.05)$ between Displays for the three middle SOAs $(30,60$, and 120$)$ combined. This indicates that the suppressive effects of the metacontrast display were greater than those of the apparent-movement display at these SOAs. The analysis of mask judgments resulted in no main effect of either Display, $F(1,3)=1.09, p>.05$, $\mathrm{MSe}=.08$, or SOA, $\mathrm{F}(4,12)=1.32, \mathrm{p}>.05$, $\mathrm{MSe}=.03$, and no Display by SOA interaction, $\mathrm{F}(4,12)=1.00, \mathrm{p}>.05, \mathrm{MSe}=.03$.

Additionally, accuracy in the test-only control condition was higher than at 0 -msec SOA. Just the opposite was found by Bernstein et al. (1976). This is not due to less overall accuracy in judging the test in this study, because the accuracies for SOAs other than $0 \mathrm{msec}$ are very similar to this study and the previous one. Furthermore, the test-only accuracies

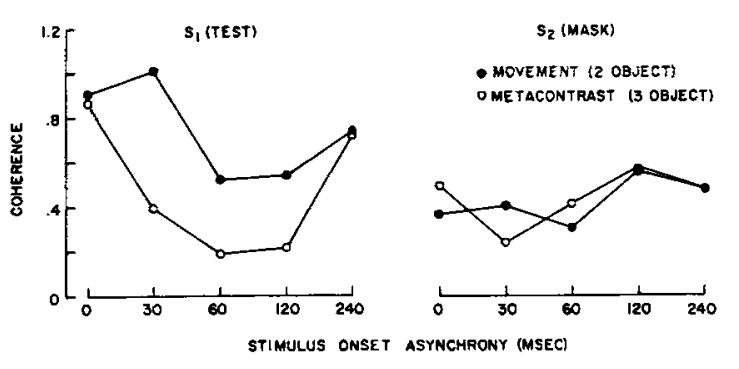

Figure 3. Coherence (expressed in terms of the quadratic form of $\mathbf{W}^{-1}$. B weighted by the LDF) for the two displays as a function of SOA for judgments of the test (left panel) and mask (right panel). 
in the two studies are quite comparable. Apparently, subjects in this experiment were merely less accurate at 0-msec SOA than subjects in Bernstein et al.'s (1976) study. Accuracy in the mask-only control condition was consistently higher than accuracies in judging the mask at the various SOAs. This indicates that there were suppressive effects of the test on the mask and that they were constant up to $240-\mathrm{msec}$ SOA. The fact that the test-only accuracy was higher than the mask-only accuracy could be attributable to the fixation circle's biasing subjects to attend to the center of the display, which is where the test was located.

The coherence data were very similar to the accuracy data. Analysis of test judgments resulted in a main effect of SOA, $F(4,12)=9.10, p<.01$, MSe $=.05$, no main effect of Display, $F(1,3)=$ $5.15, \mathrm{p}>.05, \mathrm{MSe}=.14$, and a significant Display by SOA interaction, $F(4,12)=3.28, p<.05$, MSe $=.04$. Again, a Scheffé post hoc analysis of the three middle SOAs indicated a significant difference $(p<.01)$ between Displays. Thus, responding was more consistent in the apparent-movement condition than in the metacontrast condition at these SOAs. Analysis of mask judgments indicated that there was no main effect of either Display, $F(1,3)$ $<1.0, \mathrm{MSe}=.09$, or SOA, $\mathrm{F}(4,12)=2.02, \mathrm{p}>.05$, $\mathrm{MSe}=.04$, and no Display by SOA interaction, $\mathrm{F}(4,12)=1.01, \mathrm{p}>.05, \mathrm{MSe}=.03$.

Trend analyses were also performed on the four accuracy and the four coherence functions. These results are summarized in Table 1 . As can be seen, the four mask-judgment functions are statistically flat. However, each of the four test-judgment functions contains a significant quadratic component. Thus, in general, it can be stated that the suppressive effects of the mask on judgments of the test varied with SOA in a U-shaped fashion. Furthermore, this pattern of suppression was very similar for apparent movement and metacontrast. ${ }^{2}$ The higher-order trends are not included in Table 1 because they add little to the analysis. Specifically, the apparent cubic trends in the accuracy and coherence functions for judgments of the test made in the apparentmovement condition were not significant sources of variation, $\mathrm{Fs}(1,12)<1.0$.

Figure 4 contains the goodness-of-fit data in terms of the mean percent variance accounted for by each of the two judgmental models, nondiscriminating and discriminating. Separate analyses (2 Displays by 2 Models by 5 SOAs by 4 Subjects) were performed on test judgments and on mask judgments. The mean difference in favor of the discriminating observer model was $4 \%$ for test judgments and $5 \%$ for mask judgments, which is nearly identical to the $6 \%$ difference found by Bernstein et al. (1976). However, unlike this earlier study, none of the Fs were significant at the .05 level for test judgments, although the Display by Model interaction did approach significance, $F(1,3)=7.41, p<.10$, $\mathrm{MSe}=30$. This is apparently due to the single discrepant point at 30 -msec SOA for the nondiscriminating observer model obtained with the metacontrast display, which, in turn, arose from one subject's performance. Thus, little importance is attached to this trend. The only significant source of variation for mask judgments was the main effect of Model, $\mathrm{F}(1,3)=25.87, \mathrm{p}<.025, \mathrm{MSe}=16.67$. None of the other Fs approached the .05 level of significance. Thus, the discriminating observer model provided a better fit to the data than the nondiscriminating observer model, and this difference did not depend on either Display or SOA.

The data were also subjected to a multiple uncertainty analysis (Garner \& Morton, 1969). These results generally paralleled those previously considered. There were strong contingencies between test energy and mask judgments, between mask energy and test judgments, and between the two judgments. This implies a strong process dependence at both perceptual and response levels. However, the accuracy in judging the test was not contingent on the accuracy in judging the mask. This implies a state independence. That is, any variation in sensitivity

Table 1

Trend Analyses for Individual Masking Functions

\begin{tabular}{|c|c|c|c|c|c|c|c|c|}
\hline \multirow[b]{2}{*}{ Measure } & \multirow[b]{2}{*}{ Judgment } & \multirow[b]{2}{*}{ Display } & \multicolumn{2}{|c|}{ Main Effect of SOA } & \multicolumn{2}{|c|}{ Linear Component } & \multicolumn{2}{|c|}{ Quadratic Component } \\
\hline & & & F & MSe & $\mathrm{F}$ & $\%$ Var & $\mathrm{F}$ & $\%$ Var \\
\hline \multirow{2}{*}{ Accuracy } & Test & $\begin{array}{l}\text { Metacontrast } \\
\text { Movement }\end{array}$ & $\begin{array}{l}8.69^{* *} \\
5.87^{* *}\end{array}$ & $\begin{array}{l}.0392 \\
.0339\end{array}$ & $\begin{array}{r}<1.00 \\
2.44\end{array}$ & $\begin{array}{r}1 \\
10\end{array}$ & $\begin{array}{l}31.74^{* *} \\
12.91^{* *}\end{array}$ & $\begin{array}{l}91 \\
55\end{array}$ \\
\hline & Mask & $\begin{array}{l}\text { Metacontrast } \\
\text { Movement }\end{array}$ & $\begin{array}{r}2.10 \\
<1.00\end{array}$ & $\begin{array}{l}.0237 \\
.0304\end{array}$ & $\begin{array}{l}<1.00 \\
<1.00\end{array}$ & $\begin{array}{l}3 \\
1\end{array}$ & $\begin{array}{r}<1.00 \\
1.17\end{array}$ & $\begin{array}{r}7 \\
30\end{array}$ \\
\hline \multirow{2}{*}{ Coherence } & Test & $\begin{array}{l}\text { Metacontrast } \\
\text { Movement }\end{array}$ & $\begin{array}{l}9.86^{* *} \\
3.99 *\end{array}$ & $\begin{array}{l}.0373 \\
.0473\end{array}$ & $\begin{array}{r}<1.00 \\
2.21\end{array}$ & $\begin{array}{r}1 \\
13\end{array}$ & $\begin{array}{r}35.27^{* *} \\
7.85^{* *}\end{array}$ & $\begin{array}{l}89 \\
49\end{array}$ \\
\hline & Mask & $\begin{array}{l}\text { Metacontrast } \\
\text { Movement }\end{array}$ & $\begin{array}{l}2.33 \\
1.03\end{array}$ & $\begin{array}{l}.0268 \\
.0363\end{array}$ & $\begin{array}{l}1.17 \\
1.42\end{array}$ & $\begin{array}{l}12 \\
35\end{array}$ & $\begin{array}{l}<1.00 \\
<1.00\end{array}$ & $\begin{array}{l}2 \\
8\end{array}$ \\
\hline
\end{tabular}

Note-For main effect, $d f=4,12$; for trend components, $d f=1,12$.

${ }^{*} p<.05$

$* * p<.01$ 


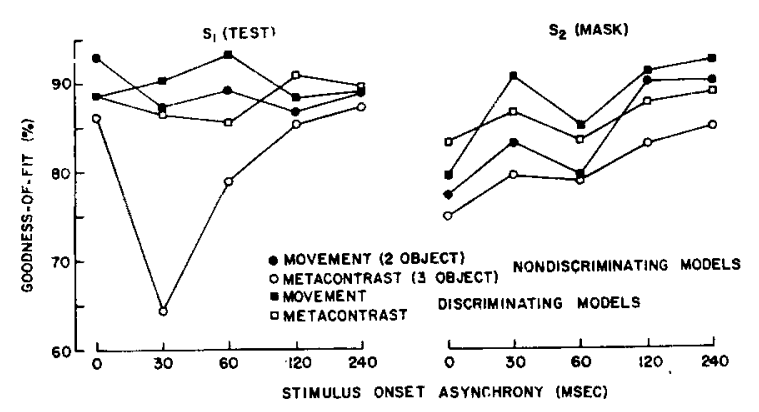

Figure 4. Goodness-of-fit (expressed in terms of the mean percent variance explained) of the two judgmental models for the two displays as a function of SOA for judgments of the test (left panel) and mask (right panel).

affecting test judgments is independent of any variation in sensitivity affecting mask judgments.

The primary results of the present study are summarized as follows: (1) Subjects responded to the test-mask energy difference at short SOAs but responded to the sum at longer SOAs; (2) this change in criterion content was present for both test and mask judgments, and no significant difference was observed for apparent movement vs. metacontrast; (3) accuracy and coherence functions for mask judgments were statistically flat across SOA and did not differ for apparent movement vs. metacontrast; (4) accuracy and coherence in judging the test were $U$-shaped functions of SOA, but the metacontrast display produced stronger suppression than the apparent-movement display; and (5) the difference in goodness-of-fit favoring the discriminating observer model was of approximately the same magnitude as previously obtained.

\section{DISCUSSION}

The change in criterion content from judgments based on the test-mask energy difference (negative correlation) to judgments based on the test-mask energy sum (positive correlation) was previousty obtained by Bernstein et al. (1976). The present study extends these findings (1) to mask judgments as well as test judgments, and (2) to suppression arising from an apparent-movement display as well as a metacontrast display.

The negative correlation found at short SOAs is explicable in terms of lateral-inhibitory processes (Breitmeyer \& Ganz, 1976; Weisstein, 1968, 1972). Thus, contrary to Weisstein and Growney's (1969) contention, the present data suggest that if inhibition is involved in metacontrast suppression then it is also involved in apparent movement.

The positive correlation found at longer SOAs is clearly not explicable in terms of lateral inhibition (as previously noted by Bernstein et al., 1976). ${ }^{3}$
However, properties of the single-unit detectors postulated by Weisstein and Growney (1969) to account for apparent movement are consistent with these positive correlations. This is because they are assumed to fire when two short bursts of energy are summed. But again, the present data indicate that if this type of detector does exist, its activation is not specific to an apparent-movement display, but rather is common to both apparent-movement and metacontrast displays.

The explanations offered by Bernstein et al. (1976) for the positive correlation between mask energy and test judgments found at long SOAs were based on the following assumptions: (1) that the judgment of the test is not an instantaneous process; and (2) that during this time the mask interacts with the decaying trace of the test, resulting in confusion as to the locus of stimulus change. Two possible sources of this confusion were proposed: intraocular light scatter and apparent movement between task and mask. These explanations can also be invoked to account for the positive correlation between test energy and mask judgments found in the present experiment.

The finding that both apparent movement and metacontrast resulted in U-shaped accuracy and coherence functions for judgments of the test does not replicate Weisstein and Growney's (1969) results obtained from the conditions in their study (Experiment 4) which most closely resemble the present conditions (i.e., monoptic viewing with a 50-msec exposure duration and essentially no intercontour distance between test and mask). The metacontrast function is best described as U-shaped, while the apparent-motion function is more nearly $\mathrm{J}$-shaped, indicating that the amount of suppression decreased at long test-mask asynchronies but that the "goodness" of movement did not.

The discrepancy between Weisstein and Growney's (1969) results and the present findings can be reconciled in the following manner. It is possible that at long SOAs the apparent movement between test and mask is still very "good" but that there is a longer delay in the initiation of this movement. This increased delay could allow additional time to abstract and process information about the test, resulting in less suppression.

As previously noted, Weisstein and Growney (1969) also found that, as the intercontour distance between task and mask was increased, the metacontrast functions decreased in amplitude (i.e., less suppression) and became flatter, but the apparentmovement functions did not change in either magnitude or shape. It is possible that, as intercontour distance is increased, the "goodness" of movement between test and mask remains invariant, but the test must "travel farther" before interacting with the mask. Again, this could allow additional processing time, resulting in less suppression. 
The previous discussion of the Weisstein and Growney (1969) results should not be regarded as a criticism of their desire to measure the appearance of motion. In fact, we did not obtain such measures because we accept their results as being an accurate representation of subjects' perceptions. We merely suggest that the fact that "goodness" of movement and "effectiveness" of metacontrast do not follow identical spatiotemporal patterns does not preclude the possibility that they are related. This is because additional factors may be involved, one being processing time.

Specifically, we propose that movement between test and mask causes confusion to the subject in attempting to extract information from the test: the better the movement the more confusion. However, some of the confusion may be avoided if there is sufficient time to process information contained in the test before the mask arrives and initiates movement. Thus, at short SOAs, there is little suppression because there is little movement. At intermediate SOAs, there is much suppression because the movement is extremely good and there is relatively little opportunity to process information contained in the test before the mask arrives. At long SOAs, there is little suppression even though the movement is still very good because the delay in the arrival of the mask affords ample time to process information about the test.

The fact that greater suppression was found in the metacontrast situation at the three middle SOAs does not contradict the assertion that apparent movement and metacontrast suppression are related. This is because one would expect increased suppression (1) as the size of the mask is increased up to some point, and (2) as the amount of contour shared by test and mask is increased. Additionally, it should be reiterated that Kahneman's (1967) formulation was based on the contention that metacontrast suppression results from anomalous or impossible motion. Clearly, it is possible that more suppression would be observed under these conditions.

The combined results of the Breitmeyer et al. (1974) study and the present experiment indicate the following: (1) When subjects are required to make the same judgment about some test stimulus under conditions which are conducive to apparent movement vs. those which produce metacontrast suppression, similar U-shaped masking functions are obtained; and (2) this is evident for both contour judgments and brightness judgments. Hence, the implication is that apparent movement and metacontrast suppression may be governed by the same mechanisms regardless of the type of judgment required as long as the subject's task remains invariant across conditions.

Additionally, the results presently obtained for brightness judgments of the mask can be compared to results reported by Breitmeyer et al. (1976). They found that accuracy in judging the presence or absence of a contour deletion in the second stimulus (mask) did not vary with SOA, thus paralleling the present finding that both accuracy and coherence functions for brightness judgments of the mask were flat across SOA. Had Breitmeyer et al. (1976) employed a metacontrast display, the present results suggest that accuracy in identifying the mask would also have remained invariant with changes in SOA.

In general, the results of the present experiment support Bernstein et al.'s (1976) finding that subjects' behavior is better described by the discriminating observer model. That is, information about the test and information about the mask are encoded separately at one level and combined at a second, higher level to be classified along a decisional axis. This is consistent with the notion that metacontrast suppression involves both sensory and cognitive factors (Bernstein, Proctor, Belcher, \& Schurman, 1974). It should be noted, though, that the nondiscriminating observer model did provide a very good fit to the subjects' response distributions and employs fewer free parameters. Thus, the assumption of a single stage results in an excellent representation of subjects' judgmental behavior.

\section{REFERENCES}

Bernstein, I. H., Fisicaro, S. A., \& Fox, J. A. Metacontrast suppression and criterion content: A discriminant function analysis. Perception \& Psychophysics, 1976, 20, 198-204.

Bernstein, I. H., Proctor, R. W., Belcher, J., \& Schurman, D. L. An analysis of U-shaped metacontrast. Perception \& Psychophysics, 1974, 16, 329-336.

Breitmeyer, B., Battaglia, F., \& Weber, C. U-shaped backward contour masking during stroboscopic motion. Journal of Experimental Psychology: Human Perception and Performance, 1976, 2, 167-173.

BrEITMEYER, B., \& GANZ, L. Implications of sustained and transient channels for theories of visual pattern masking, saccadic suppression, and information processing. Psychological Review, 1976, 83, 1-36.

Breitmeyer, B., Love, R., \& Wepman, B. Contour suppression during stroboscopic motion and metacontrast. Vision Research, 1974, 14, 1451-1456.

Garner, W. R., \& Morton, J. Perceptual independence: Definitions, models, and experimental paradigms. Psychological Bulletin, 1969, 72, 233-259.

Hake, H. W., Faust, G. W., McIntyre, J. S., \& Murray, H. G. Relational perception and modes of perceiver operation. Perception and Psychophysics, 1967, 2, 469-478.

Hake, H. W., Rodwan, A. S., \& Weintraub, D. J. Noise reduction in perception. In $K$. R. Hammond (Ed.), The psychology of Egon Brunswick. New York: Holt, Rinehart, \& Winston, 1966.

KahNeman, D. An onset-onset law for one case of apparent motion and metacontrast. Perception \& Psychophysics, $1967,2,577-584$.

Kahneman, D. Method, findings, and theory in studies of visual masking. Psychological Review, 1968, 70, 404-425.

Rodwan, A. S., \& HaKe, H. W. The discriminant function as 
a model for perception. American Journal of Psychology, 1964. 77, 380-392.

Weisstein, N. A Rashevsky-Landahl neural net: Simulation of metacontrast. Psychological Review, 1968, 75, 494-521.

Weisstein, N. Metacontrast. In D. Jameson \& L. M. Hurvich (Eds.), Handbook of sensory physiology (Vol. 7, part 4, Visual psychophysics). New York: Springer-Verlag, 1972.

Weisstein, N., \& GRowney, R. L. Apparent movement and metacontrast: A note on Kahneman's formulation. Perception \& Psychophysics, 1969, 5, 321-328.

Weissrein, N., Ozog, G., \& Szoc, R. A comparison and elaboration of two models of metacontrast. Psychological Review, 1975, 82, 325-343.

\section{NOTES}

1. This manner of constructing the stimuli may be construed as being a quantitative variation in the number of masking stimuli.
However, we view this manipulation as being qualitative in nature. That is, the two-object apparent-movement display produces "possible" movement, while the three-object metacontrast display produces "impossible" movement. Noting this, it seems reasonable to assume that accuracy, coherence, and/or criterion content of judgments produced by the two displays might not exhibit identical temporal patterns.

2. Even though movement ratings were not required of subjects, they voluntarily reported no movement at the shortest SOA and some movement at the longest SOA, but appreciable movement at the three intermediate SOAs for both two-object and threeobject displays.

3. While this does not necessarily imply a complete rejection of theories based on inhibitory processes, it does indicate that in their present state these formulations are probably inadequate.

(Received for publication July 27, 1977; 